\title{
"Alexa is a Toy": Exploring Older Adults' Reasons for Using, Limiting, and Abandoning Echo
}

\author{
Milka Trajkova \\ Indiana University, IUPUI \\ Indianapolis, IN, United States \\ milktraj@iu.edu
}

\begin{abstract}
Intelligent voice assistants (IVAs) have the potential to support older adults' independent living. However, despite a growing body of research focusing on IVA use, we know little about why older adults become IVA non-users. This paper examines the reasons older adults use, limit, and abandon IVAs (i.e., Amazon Echo) in their homes. We conducted eight focus groups, with 38 older adults residing in a Life Plan Community. Thirty-six participants owned an Echo for at least a year, and two were considering adoption. Over time, most participants became non-users due to their difficulty finding valuable uses, beliefs associated with ability and IVA use, or challenges with use in shared spaces. However, we also found that participants saw the potential for future IVA support. We contribute a better understanding of the reasons older adults do not engage with IVAs and how IVAs might better support aging and independent living in the future.
\end{abstract}

\section{Author Keywords}

Older adults; Voice assistants; Technology Non-use; Smart Environments; Life Plan Community; Focus Group

\section{CSS Concepts}

-Human-centered computing Human computer interaction (HCI) Ubiquitous and mobile computing $\sim$ Ubiquitous and mobile devices $\sim$ Personal digital assistants; Empirical studies in $\mathrm{HCI} \bullet$ Social and professional topics $\sim$ User characteristics $\sim$ Age $\sim$ Seniors

\section{INTRODUCTION}

Intelligent voice assistants (IVAs) or smart speakers, such as Amazon Echo or Google Home, provide potentially new ways for older adults to complete tasks in their homes. IVAs allow users to speak queries or commands and respond by providing an answer or completing a task on the users' behalf. In the past few years, IVA adoption has grown [36], and so has curiosity in how people are using these devices. Studies examining smart speakers use in the home $[28,39,43]$ as well as potential future uses of smart speakers $[8,27,41]$ have helped researchers to identify what

\footnotetext{
Permission to make digital or hard copies of all or part of this work for personal or classroom use is granted without fee provided that copies are not made or distributed for profit or commercial advantage and that copies bear this notice and the full citation on the first page. Copyrights for components of this work owned by other than the author(s) must be honored. Abstracting with credit is permitted. To copy otherwise, or republish, to post on servers or to redistribute to lists, requires prior specific permission and/or a fee. Request permissions from Permissions@acm.org. CHI 2020, April 25-30, 2020, Honolulu, HI, USA.

C 2020 Copyright is held by the owner/author(s). Publication rights licensed to ACM. ACM 978-1-4503-6708-0/20/04 _.\$15.00

DOI: https://doi.org/10.1145/3313831.3376760
}

\author{
Aqueasha Martin-Hammond \\ Indiana University, IUPUI \\ Indianapolis, IN, United States \\ aqumarti@iupui.edu
}

users perceive as benefits and barriers to smart speaker adoption. There has also been growing interest in how people with disabilities [1,39] use smart speakers and emerging interest in smart speaker use among older adults $[8,40]$. These studies have helped to advance knowledge of use, but also awareness of open IVA design challenges and potential future uses. However, despite the growing interest in smart speaker use in the home, we know little about the complementary perspectives of IVA non-users, particularly older adults. Studying IVA non-use can bring different perspectives about real-world use [42], but also help further advance understanding of how to improve design.

In this paper, we explore IVA non-use or reasons why older adults limit or abandon smart speakers through the lens of a group of older adults that are members of a multi-site Life Plan Community (LPC) in Maryland, Washington D.C., and Virginia. We conducted eight focus groups with a total of 38 participants 65 years of age or older that resided at three different campuses supported by the LPC organization. Thirty-six of our participants were volunteers of an early adopters' program for Amazon Echo, a voiceonly intelligent smart speaker, provided and supported by the LPC. Two participants had not adopted Echo but joined the focus groups to share their concerns. At the time of the study, 36 participants had the Echo device in their homes for at least one year. All participants of the early adopters' program joined with the initial intent of using Echo, and many were also active members of a pilot group that was to test new Alexa skills (i.e., software) for the LPC.

We found that a year after the Echo was set up in their homes, many participants had limited or abandoned use of their Echo device. Participants described four categories of use in focus groups: active use, limited use, abandonment, and lagging adoption. However, the majority of participants described types of non-use (limited use, abandonment, or lagging adoption). Participants struggled to find what they considered valuable use cases, questioned the value of IVA use based on their beliefs about their abilities, and encountered challenges negotiating use in a shared home environment. Despite their perceptions of Echo as nonessential in their current situation, most participants could see how existing and new IVA applications might benefit them or the LPC in the future. We contribute a better understanding of the reasons why older adults do not engage with IVAs and opportunities for IVA applications to better support aging and independent living. 


\section{RELATED WORK}

We discuss literature related to IVAs design and use by varying user populations, voice-based smart home interfaces for aging, and studying non-use in HCI.

\section{Examining Voice Assistants Use and Design}

In the last few years, there has been increased interest in understanding how people adopt and use intelligent voice assistants (IVAs). In 2017, almost $46 \%$ of the U.S. population used an IVA, such as Siri or Cortana [36]. Currently, most IVAs are used to support basic, daily tasks through voice-based interactions. While dedicated IVAs such as Google Home or Amazon Echo provide somewhat different affordances and have lower adoption rates [36], they share some of the same interaction challenges as mobile IVAs such as Siri. Therefore, we discuss both.

Studies have found that individuals that adopt IVAs find them useful for entertainment, basic queries, and smart home control $[28,43]$ and for supporting these tasks among older adults $[8,23,40,41,49]$ and individuals with disabilities [1,39]. Sciutio and colleagues, for example, studied how people use IVAs but also their purchasing practices, placement, conversational patterns, and how children interact with IVAs in the home [43]. Pradhan and colleagues studied IVA use among individuals with vision impairments and found similar uses and placement, but also examined factors influencing the accessibility of the device [39]. Researchers have also explored IVA use in public places and how the use of voice-controlled devices impact users $[17,27,49]$. These studies have contributed a better understanding of IVA use but have also uncovered barriers.

Barriers to use and adoption are common findings among studies of IVA use. User perceptions about the utility and privacy risks associated with IVAs are known to influence adoption [20,25]. Once adopted, users sometimes assign "human-like" qualities to their device [29] and thereby, establish greater expectations for interaction [13,29] and conversation [9] that are not met by the device. In addition, privacy concerns continue to persist after adoption, causing some to alter their use $[13,17,26,30]$. Identifying barriers to IVA use and adoption help designers better understand the challenges that negatively impact use.

\section{Smart Homes and Voice Interfaces for Aging in Place}

While some older adults desire to maintain an independent lifestyle for as long as possible in their homes, they must leave their homes much sooner than they prefer due to health and safety risks [46]. There are known tradeoffs of adopting smart home applications [7], such as an IVA. Still, for years, these applications have been seen as an opportunity to support independent living among older adults while also addressing safety concerns [12,15,16]. However, older adults have had mixed views about smart home technologies, especially voice-based applications.

Some older adults tend to find aspects of voice interfaces useful for supporting independent living through simplified interactions, which can be potentially helpful for older adults with limited technical experience [49]. On the other hand, Portet and colleagues found that while older adults appreciate the possibility of using voice for home automation, they also fear that voice-controlled devices make them more dependent [38]. Vacher and colleagues also found that older adults appreciated voice control for risky or complex situations, but preferred manual interactions for home automation because they viewed it as more efficient [47]. Lucia and colleagues found that older adults saw voice-based interfaces in smart homes to be a tradeoff to other interfaces they had used that provided more situational awareness [31]. Therefore, while older adults see the benefits of using voice-control in smart home environments, there are still open concerns and design challenges that impact older adults' adoption and use of these technologies in their homes.

\section{Studying Non-use in $\mathrm{HCl}$}

While past work has led to a significant understanding of IVA use and barriers, with the exception of a few studies $[13,30]$, most are framed from the perspective of understanding use opposed to non-use. Studying technology non-use contributes additional understanding of use, particularly in real-world settings [42]. Existing technology acceptance models such as TAM [14] and UTAT [48], primarily focus on usage but have been used to study nonuse. For example, Neves and colleagues used TAM both directly [35] and indirectly [34] to examine technology nonusage among older adults. However, these models have also received criticisms because of their limited focus [2].

The study of technology non-use takes a more intentional approach of examining more broadly the reasons current and former users [50] and early adopters [44] limit or abandon technology regardless of their intention. While there has been limited focus on IVA non-use among older adults, there is significant literature outlining the importance of the intentional study of technology non-use in different contexts $[3,44]$. Studies of Facebook non-use have led to better understandings of when, how, and why different types of non-users leave Facebook [3,19]. Studies have also examined abandonment of self-tracking tools [18], wearables [10], and other smart devices [24].

While technology non-use suggests the absence of use, defining non-use can be complex. Existing classifications of non-use lie on a continuum between use and non-use, considering that non-users may engage with the technology at some level. For example, Wyatt and colleagues classify non-users as those that resist (actively choose not to engage), reject (engage initially, but abandon), are excluded (ostracized), or are expelled [50]. Satchell and Dourish describe non-use in terms of lagging adoption, active resistance, disenfranchisement, displacement, disinterest, and disenchantment [42]. Baumer introduces the term Usees to describe a form of use between use and non-use that do not fit established definitions in either category[3]. 
However, despite the varied definitions of non-use, there is consensus that examining non-use is essential for understanding use and can help improve design. We extend prior work by examining reasons that older adults limit, lag in adopting, and abandon IVAs (i.e., an Echo device). Our work examines older adults' reasons for IVA non-use after an extended period of use (at least one year). To our knowledge, we are also the first to examine IVA use and non-use in an independent living environment.

\section{METHODS}

We conducted eight focus groups with members and aspiring members of an early adopters' Amazon Echo program offered at a multi-site Life Plan Community (LPC). The purpose of this research was to understand the reasons older adults use, limit, and abandon Echo in their homes. We addressed the following research questions:

RQ1: How are older adults using Echo in their homes and to connect with the LPC community?

RQ2: Why do older adults limit and abandon Echo?

RQ3: In what ways do older adults perceive IVAs can provide value in the future individually or at an LPC?

Using focus groups provided advantages for capitalizing on the dialogue between participants to reveal insights and perspectives about Echo use both as individuals and as LPC members [6,22]. Our study design is inspired by past studies that assign [45] or assist users with the purchase [24] of technology to explore non-use. However, we retrospectively examined a real-world deployment.

\section{Recruitment Site and the Early Adopters' Program}

Life Plan Communities (LPC), formerly known as Continuing Care Retirement Communities (CCRC), offer a full continuum of care, from independent living options such as free-standing homes to 24-hour nursing services and memory support, all on one convenient campus. An LPC often provides maintenance-free living for residents with services and amenities such as housekeeping, scheduled transportation, social activities, and dining. Participants in our study lived in an LPC that emphasizes holistic wellness through active, engaged, and healthy lifestyles, social responsibility, and different levels of care. The LPC includes several locations throughout Maryland, Washington D.C., and Virginia, and participants in our study lived independently in homes in one of the three sites.

The Amazon Echo early adopters' program was funded internally by the LPC because of leadership and residents' interest in Echo. The LPC found that $30 \%$ of its members were unable to connect with information and residents/staff due to disabilities. The LPC initially, therefore, became interested in IVAs as an alternative method to improve the social, physical, and cognitive well-being of residents. However, participants of the program were all volunteers with interest in exploring Echo use for different reasons (e.g., accessibility, entertainment, finding information). A prior survey also revealed that the majority of the participants in the program had positive attitudes towards computer use and its perceived utility.

\section{Participants}
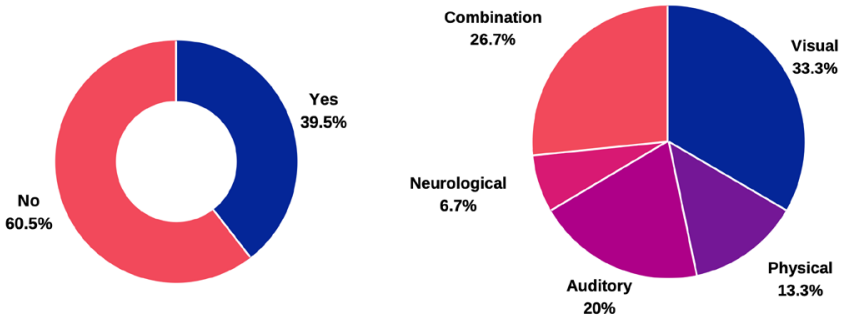

Figure 1. Participants' self-reported disability status. Visual disabilities $(33.3 \%)$ were the most reported disability followed by more than one $(26.7 \%)$, auditory $(20 \%)$, physical $(13.3 \%)$ and neurological (6.7\%) disabilities.

Fifty percent of participants self-identified as female and fifty percent self-identified as male. Most participants were retired $(\mathrm{N}=33)$ and lived in two-person households $(57.9 \%)$. All others lived alone (42.1\%). Of the 38 participants, fifteen participants self-reported a disability or impairment (See Fig. 1). Education level varied; however, most participants had at least a Master's degree $(\mathrm{N}=18)$. Most annual household incomes were over $\$ 60,000$ (USD), out of which $58 \%$ were over $\$ 90,000$ (USD). Participants volunteered for the study and did not receive an incentive.

\section{Focus Group Sessions}

We conducted eight focus groups across three different sites within the LPC. Our study was reviewed and approved by the Institutional Review Board (IRB) at Indiana University before any data was collected. We collaborated with staff at the LPC to recruit participants, organize focus groups on site, and coordinate travel to each site. The LPC staff assisted in screening participants to ensure that participants owned an Echo for at least a year and scheduling participant sessions. All focus groups were conducted in a private room at the LPC to protect participants' privacy. To mitigate conflicts of interest, the LPC staff did not participate in the development of the study protocols. On the day of the study, LPC staff assisted researchers and participants with gaining access to rooms in the facility but did not participate in facilitating focus groups. We also collected consent documents that provided detail about the study, its voluntary nature, and participants' rights regarding their protected health information. We also shared that we were not acting on behalf of the LPC and that data collected would be reported anonymously. 


\section{Experiences with Echo}

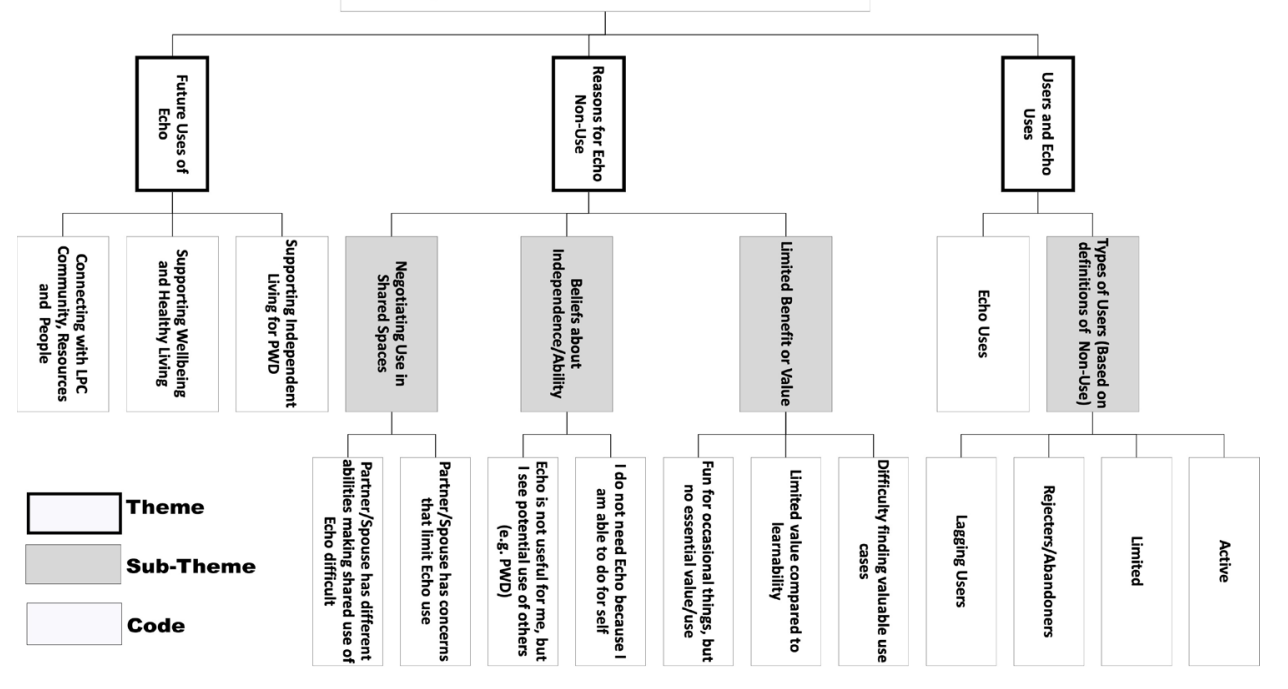

Before beginning the discussion, we asked participants to complete a demographic and background survey. We then prepped participants on focus group etiquette and answered questions they had about the study. During the focus group session, we asked participants about their reasons for joining an LPC, how they used their Echo device(s), reasons for use and non-use, benefits and challenges of adopting Echo, and how they believed that Echo or a similar device might benefit them in the future. We also discussed the challenges they had in the LPC to understand other ways IVAs might help them in connecting with the broader community. Each focus group lasted about an hour, and with permission, we audio-recorded each session.

\section{Data Analysis}

We transcribed and analyzed audio recordings from each of the eight focus group sessions using inductive and deductive coding [11]. Initially, two members of the research team read each of the focus group transcripts independently and made marginal notes representing an initial set of emerging codes. We did not use any existing acceptance models as framing and instead used an inductive approach guided by $[11,32]$ to understand usage more broadly. Researchers met several times to iteratively compare, discuss, and reconcile codes [32]. From this process, we developed an initial codebook representing participants' experiences with Echo, resulting in three themes: Users and Echo Uses, Reasons for Echo Non-Use, and Future Uses of Echo (See Fig. 2).

We re-coded each transcript, deductively assigning codes from the codebook to blocks of text. We also used Wyatt's [50] and Satchell's [42] definitions of use and non-use types to categorize participants based on their descriptions of Echo use. This categorization resulted in four additional codes: active users, limited users, rejecters/abandoners, and lagging users. Analysis resulted in fifteen codes, four subthemes, and three themes (See Fig. 2).

\section{FINDINGS}

We report the types of Echo users present in our study and overarching themes regarding use, non-use, and potential future uses of Echo discussed in the focus group sessions.

\section{Types of Users and Echo Uses}

Interview participants represented several different types of Echo users, including active users, limited users, rejecters/abandoners, and lagging adopters $[42,50]$. Several participants self-described as non-users of Echo, but most described using Echo at some level frequently or in a limited capacity. In non-use literature, it is common for a user to use technology at some level, but be considered a type of non-user $[3,42]$. For example, limiting and relapsing (abandoning and returning) are both considered valid types non-use [4]. Most participants usage ranged somewhere between use and non-use, with fewer at the extremes.

Overall, seven participants $(\sim 18 \%)$ described using their Echo device daily (active users). Participants in this category expressed that they used Echo frequently for daily tasks, enjoyed using Echo, and sometimes purchased additional devices because of the value it provided.

"So, I got my Alexa. I set it up. I use it every day. I use it for my calendar, the weather, for alarms and timers, and I use it for my grocery list. And every now and then, I'll use it for some off-the-wall thing." PFG7

Five participants $(\sim 13 \%)$ had abandoned the Echo device and discontinued use (rejecters/abandoners). These participants explained that after initial exploration and use of their Echo device, they found no value in its features, and therefore, stopped using it or gave it away.

"I used to have an Alexa two years ago, but it was so limited use that I gave it away." PFG4

Twenty-four ( $\sim 63 \%)$ participants described limited use of the Echo, noting that they only used it minimally. Most of 
these participants self-described their use as infrequent or occasional. However, a few self-described themselves as non-users, but mentioned "playing" with the device.

"I use Alexa also very minimally. My best use of it is to get music and all I have to do is to give certain commands ... and I use consistently the same commands so that she knows exactly what I mean. ”, PFG3

Two users $(\sim 5 \%)$ were lagging adopters that had interest in Echo and joined the focus group to learn and share their concerns for adoption. These participants were not part of the initial pilot program and had yet to adopt Echo because of existing concerns regarding the device.

Table 1 lists the most commonly discussed (mentioned) uses of Echo by participants. Among the most frequent uses was listening to music, setting alarms/timers, checking the time, and checking the weather forecast. Both users and non-users of Echo described using their Echo frequently to listen to music for entertainment, wellness, and relaxation. Participants also frequently used Echo to set alarms for sleeping, cooking, and appointments. Less frequently, participants used Echo for news, jokes/games, finding answers, and their calendar. Other uses mentioned once were spelling, lists, books, and entertaining grandkids. These findings are similar to other studies suggesting IVA use similar to that of other user populations [29,37,39].

\begin{tabular}{l|l} 
Use & Number of Participants \\
\hline Music/Radio & 16 \\
Alarm/timer/time/reminders & 10 \\
Weather & 9 \\
News & 5 \\
Jokes/games & 2 \\
Calendar & 2 \\
Dictionary & 2 \\
General knowledge & 2
\end{tabular}

Table 1. Commonly discussed Echo uses by participants.

\section{Reasons for Echo Non-Use}

Participants encountered both usability and accessibility challenges when using Echo; however reasons for non-use mainly stemmed from other factors. Participants discussed limiting or abandoning Echo due to limited benefit/value, personal beliefs about IVA use and ability, and challenges negotiating use with other household members.

"Alexa is a Toy" - Limited Benefit, Limited Value

When discussing why they limited or discontinued use of Echo, one common reason participants noted was that they found it difficult to identify use cases that provided essential benefit or value. One participant explained,

"If you're tech enough to be able to wade through that thicket to get there [Alexa setup], you can start slowly building a use case for how you might be able to use it ... I'm still in a voyage of discovery and I'm trying to figure out what are we going to use it for? Is it worth the cost? Is it worth memorizing how to get the weather when I can just look at my iPad, and tap the weather channel. That's where
I have the disconnect right now. It actually is not more convenient for me." PFG5

Participants often discussed the initial excitement of using Echo when they signed up for the Echo pilot program, but that over time they felt it was difficult to find uses for Echo that were essential to them daily. The topics of essential value or essential benefit of the Echo compared to having any value or benefit was discussed frequently among participants and across the focus groups. Most participants found uses for their Echo in one way or another and found it convenient for some tasks such as playing music. Therefore, one might infer that Echo had valuable uses; however, most participants did not view this type of use as essential and thus, at times, self-described themselves as non-users even as they described how they used their Echo.

"I ask her every morning, "What's the weather today in Maryland?"...By that time, I've brushed my teeth and I'm off for the day. That is how I use Alexa. Every once in a while, I ask it to play a little quiet piano music by Beegie Adair, but other than that, I don't use it and I never know whether she's still on when I walk away." PFG4

Because participants viewed Echo as providing limited essential value, they often compared using Echo with another medium that was more familiar to them or easier to use. In these cases, participants would choose the other medium that was easier to use and satisfied their needs for completing the same task in the past instead of using Echo.

"The only thing I use it for is occasionally to listen to Pandora music. If it had the skills that we would hope someday...that work every time you ask it instead of once every six times, I would use it. But for now, for weather, I prefer my iPhone because I can see the days ahead rather than just today. So as far as I'm concerned, it's [the Echo] is a waste today." PFG7

Some participants' beliefs about the lack of essential benefit were not limited to Echo, but also other IVAs such as Siri. They expressed that because of their ability to access similar information more quickly from other mediums, IVAs were often more of a nuisance than a help to them.

"I got Siri turned off. I've always thought it was more annoying than anything else. To me, it slows down the accessing of information. But then I've sort of lived with an iPad in my lap now for about seven years. So, it, to me, it's second nature to use an iPad." PFG5

Participants' discussions about the essential value of Echo and their ability to use other devices to complete the same tasks, led participants to often describe Echo as a "toy" that was nice to "play" with but not necessary for their daily activities. The description of Echo as a toy also seemed to explain some of their views of themselves as non-users. While participants would often describe using Echo for tasks, they would describe those tasks as non-crucial or for 
"fun", and that if the Echo was taken away, it would be easy to complete those tasks using other methods.

"From my point of view, its greatest potential is for those people with poor eyesight who need help and can communicate verbally but cannot read. That's not either of us, my wife or myself. Therefore, it is much for us, more of a toy than a useful necessity. It's not the center of any of our universe." PFG3

Participants' perceptions of Alexa as "toy" were also related to their search for valuable Echo uses. Some participants noted emails they received about potential Echo uses and their disappointment in the recommendations.

"My experience with the [Alexa suggestion emails] ... I don't get the email directly; my wife does from Amazon. When I do look at them, 99\% are silly...I would not want to waste the ink on printing it out [the suggestions]. " PFG3

Participants' beliefs and use of Echo seemed to be consistent with their views of Echo as "toy". Echo was sometimes nice to have, fun or convenient to use, but overall provided no essential value to their daily lives apart from what they were capable of doing with other more familiar mediums. Therefore, at times even though they described use of Echo, they would describe this type of use as non-use and themselves as non-users because in their opinion they had no essential need for Echo in their homes.

\section{Beliefs About Individual Ability and Independence}

Discussions about essential value and need for the Echo device were tied to some participants' beliefs about individual ability and independence. Several participants believed that the Echo device could indeed provide essential benefit to them due to a recent change in their abilities, although they shared challenges similar to others.

"I think I'm probably, and maybe one other person whose sight is as bad as mine, but I don't think anybody else is quite as bad. And it all happened in a year, so I'm learning of things that I used to do that I took for granted that I can't do anymore. I would like to be able to use Alexa for what I lost.” PFG2

Participants, both with and without disabilities, saw value in using Echo to support individuals with disabilities or future versions of themselves with reduced independence.

"The reason I even got involved in this [the pilot program] is because sometimes I may not be able to get on the computer and do things, and so I want to be knowledgeable before I have to. Because, that too might kind of go [the ability to do things independently].” PFG4

However, most participants discussed that in their current circumstance, one of their reasons for non-use related to their belief that they still had the ability to complete tasks on their own, and they enjoyed this freedom. Participant's attitudes about the use of the smart home capabilities of
Echo were that using them could negatively impact the independence of older adults.

"I wouldn't even be interested in it [Echo functionality], quite frankly. I think my view to Alexa, personally, right now, in my mental and physical state is that I find it a convenience where the music is concerned because I don't have to be playing all the discs anymore ... But I think for people who are basically independent and want to continue significant independence, I think Alexa could be an unfortunate thing, personally.", PFG8

Others described that they did not like the idea of asking an assistant to do something that they could do themselves but could understand how those features might be useful to someone with different abilities. Additionally, participants' beliefs about independence and ability were not only related to the smart home capabilities of Echo, but also to search features as some participants preferred to be more engaged in the process of finding the information.

"I don't like it [using a voice assistant]. I feel very reluctant to speak out loud to an assistant to do something. But that's maybe just personality type or something. I'm just saying around the house to ask for weather, it just bothers me to speak it out loud and then have the answer essentially spoken out loud to me, as opposed to pulling out the phone and looking it up." PFG5

Often participants would compare their need to use Echo with their physical ability to complete tasks. For example, another participant explained that because they felt their vision was not impaired and was comfortable with a computer, they preferred the computer, however, if their vision were to become impaired, they might reconsider.

"I'm on my computer most of the time. That's what I do, so that's the way I am comfortable doing things, and my vision is still good. I think if my vision declined, I would be more interested in using Alexa. Alexa does require you have to ask things exactly the right way. So, it's very difficult compared to a computer, in my mind, to use." PFG1

Most participants could see Echo's potential value; however, they often saw value not for themselves in their current situation, but for others that had different abilities, independence, or future versions of themselves. Participants who did not see Echo as essential shared reasons related to self-sufficiency and independence. Participants explained that they were still capable of completing many of the tasks Echo could do using other methods.

Negotiating Use in Shared Spaces

Another common reason for non-use was the need to negotiate Echo use in shared spaces. Negotiation often occurred between individuals living in the same residence where one person was open to using Echo, and one person was not interested. In these situations, participants would describe workarounds but eventually began to limit the use of Echo to respect the other persons' wishes. 
"Well, when I moved in my wife was here, and she died two years ago. I've done most of my Alexa since she passed, but it [Alexa] wasn't something she was excited about." PFG6

Some conversations around negotiating use were related to privacy concerns. Participants heard rumors that "Alexa is listening" or recording conversations, and it was concerning for some. Those concerned mentioned not wanting sensitive conversations recorded, but others felt it was just unethical.

"There's a lot of concern now that Alexa is listening. My husband's always been concerned, but I'm hearing it from other people. This random person came up to me and said, 'Did you hear, Alexa's listening all the time? I guess it's the principle of the thing that bothers people." PFG1

Other participants discussed that privacy and confidentiality were their primary reasons for limiting Echo.

"I guess a good part of my reason for doing less on Alexa is that I have no form of assurance that I have privacy and confidentiality when I use Alexa. There has been some information out there that questions Amazon's protection for individual privacy. So, that adds to my hesitancy of using it. And I still keep looking for some kind of definitive response from the Amazon people that will provide me with greater assurance as an individual.", PFG2

In shared spaces, the issue of privacy was also sometimes a major concern of one household member, but not as much for another. Participants, therefore, began to limit their use of Echo or the location it was used in the household.

"My wife is sufficiently concerned about its lack, or alleged lack of privacy, so that she banished it out of our bedroom and into a different room. It's in my study now, and I occasionally play with it but that's about it." PFG3

One participant's husband had similar privacy concerns, and therefore she limited her use to times he was not home.

"I haven't used it as much because my husband is really concerned about privacy, and we have a physical switch on it that's turned off, especially when he's in the apartment. And when he's not, and I know that he's not going to be around, I'll turn it on. And the thing I like the most is being able to say, "Alexa, news" and get what's going on or, "Alexa, music," and I tried to think of some kind of music that I like, and I like that." PFG1

Privacy concerns led to compromises, but varying interaction needs were also discussed. One participant described limiting his use due to his wife's anxiety. He used Echo initially but found that it frightened his wife.

"Well, I used it a little ... but one of the things is when it comes on it sort of frightens my wife, and she is apartment all the time. When she's out, I'm always with her. So, there's not a time when she's not there when I can use it ... the noise [scares her], and she does have anxiety disorder, so she's very easily gets anxious. She has a history of trauma and it's very easy for her to get upset" PFG1
Another participant wore hearing aids and found it challenging to hear Alexa. Even after adjusting the volume, there was an additional burden of negotiating use with their spouse, who had different hearing abilities.

"I'm hard of hearing. So, I have hearing aids, but I have a frequency problem, and it would be helpful if I could tune Alexa a little bit on frequency. My hearing loss is more severe in the higher frequencies than the lower. With the hearing aids, I can compensate a little bit... But you know, constantly turning it up and down for things, to make it easy for me ... because my wife has acutely good hearing. So, we're at opposite ends of the spectrum, constantly saying, "Alexa quieter." Or, "Alexa louder." is a little bit of an inconvenience." PFG5

Participants' challenges negotiating use with their spouse or partner, whether due to privacy concerns or differing interaction needs, led them to limit their use. For some, limiting resulted in the physical removal of the device from certain rooms or to times when their partner was not around. For others, they abandoned the device altogether.

\section{Future Echo Uses}

While most participants believed that Echo was not useful to them in its current state (i.e., out of the box), they described several ways Echo could be potentially helpful to them in the future, including for accessibility, well-being, and connecting with the LPC and broader community.

\section{Supporting Independent Living for People with Disabilities} As discussed earlier, one of the main potential opportunities' participants saw for using Echo or other similar voice-based devices was to support individuals with disabilities or with less mobility, such as those residing in assistive care. Similar to other studies [1,39], we found that some participants with disabilities were already using their Echo as accessibility aids. For example, one participant with impaired vision, which was characterized as an active user, described that he used his Echo device daily.

"I use it every single day. I can't see the clock anymore, so I ask Alexa the time at different times during the day. I can't read the [LPC newsletter] and so every day I check to see what the events of the day are. Sometimes I use it for other things, but that's sporadic. I also use Alexa as a reminder if I have to go someplace, I'll ask Alexa to remind me ten or fifteen minutes before I'm to leave.", PFG2

Other participants remarked how they felt Echo or a similar device might be useful in the future if they were to need assistive care or experience a change in ability. Therefore, while most participants currently felt Echo was not useful, they saw how it or a similar device could be useful in the future if they experienced changes in their independence.

\section{Supporting Wellbeing and Healthy Living}

Participants also saw potential uses for Echo to assist them and others with daily health and well-being tasks. While currently, IVA devices have limited focus on health and 
wellness, participants saw opportunities to use existing features or to create new features that support them individually at home or in the LPC community with health and wellness. Several participants were already using their Echo's music features for wellness purposes.

"One of the ones I really liked was Healing Tunes. Just soft music in the background, when you're relaxing and you're resting and... Having moved here this last summer when I needed to have about ten minutes out, it was wonderful to have those healing sounds. " PFG6

Using IVAs for music is common; however, participants suggested that this feature could be scaled in the LPC to support wellness activities in individual homes and LPC units. One participant shared her experience when visiting the nursing unit for a broken leg. The unit used Echo's music features to support patients as they healed.

"They use Alexa, well, little Echo Dots over in the nursing center, to play music for the people over there. Especially the music that they had as young people. And it's amazing. There's a video that talks about how people that have been in comas kind of wake up and come out of it and they start interacting because the music got through to them." PFG6

Another participant described how a different unit was using Echoes to support those with mental impairments. Patients in this unit lived in a controlled environment with limited access. However, the LPC created an outdoor environment to support the patients' wellness needs.

"I have enjoyed seeing it [the Echo] being used in the secret garden ... and it's remarkable with that group of people what it can do for them. But they are somewhat interacting with what Alexa can do ... music, stories. So, they go out and sit in the secret garden, and ask the machine to perform ... The Secret garden is a place that is walled off for memory impaired people. It's an outdoor area that has plants ... Gives them another environment to interact with. They get outside in the sunlight." PFG7

While not official, there were emerging efforts throughout the community to explore using Echoes and similar devices to support individual and community health and wellness efforts. Participants saw these efforts as good potential uses for Echo or another IVA. For individual use, participants discussed how Echo might assist them at home with daily wellbeing activities such as tracking their health indicators.

"If you interact socially at our age, you actually prolong your life. It's a healthy thing. Alexa gives some of our more isolated folks an ability to do that. If you just know your metrics, you get healthier because...you start paying attention to them...your blood pressure drops, your weight drops. Alexa [we hope] will help us do all of that." PFG7

One practical use participants discussed was the ability for Echo to assist them with wellbeing checks and alerts. Each independent living residence has a bathroom button to alert staff of an emergency. The button must be pressed each morning to avoid a well-being check from staff. Participants felt that a voice-based system such as Echo would be potentially useful for facilitating these checks and other emergency calls to LPC staff.

"Can we just say 'Alexa, push the button?' It would be nice, because, if you have an incident and you fall... are you going to be able to crawl to the bathroom and reach up on the metal lever about four feet up to pull the cord. It would be nice if Alexa could do something to help in that for emergency situations. " PFG5

In addition to individual health needs, participants discussed opportunities for Echo to assist them with daily caregiving tasks.

"We also have a lot of needs where there are couples, one being a caregiver for either medical need for their partner, or medical and memory, and cognitive, and in those cases, caregivers could use all kinds of good health help, and Alexa I think can be there in a variety of ways to help with entertainment but also information and companionship. All those things are needed every day. " PFG5

Most participants mentioned that in addition to being an accessibility aid, they saw the potential for Echo to provide "good health help" as an entertainer, companion, and information source. Participants noted that some features were currently available in IVAs, but they preferred tailored applications both individually and within the LPC.

Connecting with the Community, Resources, and Others

Many participants discussed that one of their reasons for joining the pilot program was to explore ways the Echo could help them better connect to other residents, the LPC community, and the resources provided. Most participants described themselves as highly active and, although retired, were busy using their prior experiences to bring together people in the LPC and the local community.

"That's a common thing we run into is that you think when you retire you get a little more time. You don't. You're much more busy. At the same time, our kids think we just sit around, so we get these toys. " PFG7

Participants often mentioned the importance of community when describing reasons for joining an LPC. Apart from financial considerations, whether they joined the LPC due to health decline, personal injury to themselves or a spouse, or to lessen the burden on their children, they prioritized having a sense of community when choosing their next home. While the community had some of these mechanisms in place, participants saw Echo as a potential tool for improving how they engage with others and resources available to them in the LPC. Several participants discussed their desire for Echo to help them make phone calls.

"I had the impression that you are not supposed to be using the [LPC] contact list by contacting Alexa. But if you can, that a big advantage. Primarily, if you don't have it 
memorized, the phone number, you either have to go look in the portal for the resident directory. " PFG8

Others described desires to connect with volunteers and other LPC members willing to assist them with tasks in their homes. One participant with vision impairments described his current challenges finding volunteers.

"I haven't had anybody [a volunteer] come consistently, but I'm trying. Part of my problem is I can't read the book with the volunteer's name. So, it's all by word of mouth and asking people.” PFG2

Another participant replied that finding volunteers would be a good opportunity for Echo to connect them with others willing to help with tasks in their home.

“... [the] volunteer or Neighbor to Neighbor Program, it's actually ideal for to be used with Alexa because it's fairly structured, yeah? I need access to volunteer. What do you need? You know, so it's fairly structured.” PFG2

Participants saw the most value in an IVAs' for use as a practical tool that could help them more easily stay informed about people and things they cared for and valued.

"Perhaps, what people or I would need, is some more practical uses. A list of more practical uses for the Alexa, as opposed to what they [Amazon] have prescribed." PFG8

Participants suggested other features that would enable access to exchange programs for assistive devices (e.g., wheelchairs) in which they could connect to people that had available devices or to find out about available devices in the community. Others discussed being able to learn about community events and groups through an Alexa skill. Therefore, participants most valued skills that would support community building inside and outside of the LPC.

\section{DISCUSSION}

Our findings highlight older adult participants' experiences adopting Echo while living in an LPC environment and the subsequent challenges they encountered identifying essential use cases in consideration of their beliefs about independence, ability, and other people living in their homes. While many participants reported using Echo for tasks (e.g., music) that are common of IVA users, most did not associate this as essential use and thereby self-described as non-users. Among the types of non-users described by Satchell and Dourish [42] are those that actively resist adopting new technologies for one reason or another. These users choose not to engage or use a new technology. However, most of our participants did not fit this category of non-use and, despite currently limiting and abandoning Echo, saw potential for future use of Echo and other IVAs. We, therefore, contribute additional insight into why older users limit or abandon IVA devices over time and how they believe IVAs might be useful to them in the future. With the exception of a few studies such as Luger's work on conversational agents $[13,30]$, there has been limited focus on reasons for IVA non-use. We discuss our findings in relation to prior work on IVAs and other conversational interfaces as well as highlight, based on our findings, opportunities to improve IVA interactions and use among older adults and in independent living settings.

\section{Essential versus Casual Use Cases}

Like Luger and colleagues' study of conversational agent non-use, we found that our participants felt that Echo was nice for entertainment purposes, and many initially engaged in playful activities to learn more about what Echo and Alexa could do for them [30]. However, unlike some participants of that study, few of our participants at the time were willing to invest time in exploring or discovering new use cases that they felt might be more valuable or beneficial to them. While some participants mentioned the need to learn more about what Echo could do, many also mentioned lack of time, support, and desire to find essential use cases as a limiting factor to their Echo use.

Distinguishing essential use from casual use was important to uncovering our participants' beliefs and needs regarding Echo. The role of benefit on older adults' motivation to adopt new technologies is well known and is found to be important regardless of their decision [33]. While attitudes about Echo were positive in the beginning, over time, participants' views of “Alexa [the Echo] as a toy" emerged in part, from their inability to find essential utility in comparison to what was already available to them. Thus, interest waned, and they placed less value in attempting to use the Echo. These findings relate to Han and colleagues' findings that a user's belief about ease of use and utility of an IVA for a task relates to user satisfaction [20]. These findings also align with existing technology adoption models that emphasize perceived usefulness as a factor in adoption $[14,34,48]$. Prior work has identified a need to better support exploration and discovery in IVAs $[30,39,43]$. However, for older adults, particularly those that might not see an immediate need for an IVA identifying and distinguishing essential opposed to casual use cases might lead to broader adoption in the future.

\section{Ability and Independence Beliefs versus IVA Use}

Because participants did not find essential utility in consideration of their abilities, similar to other studies, they often opted to complete tasks in ways that they felt were easier, more efficient and aligned with their beliefs about independence $[31,38,47]$. However, our participants' attitudes about independence and ability as it relates to Echo use was somewhat contradictory to what has been found in other studies of IVA use with similar groups. Many participants openly questioned the use of Echo and related use to loss of independence or "laziness", although they often described using a computer or other technology to complete similar tasks. Others noted that they viewed themselves as independent and self-sufficient and therefore preferred completing the tasks that the Echo might complete on their own. Luria and colleagues found that voice-based interfaces can often lead to user perceptions of 
less control over the device [31]. Therefore, our participants could have viewed interactions with Echo as providing less control over tasks that they highly valued or appreciated than with other devices. However, participants' beliefs about independence and Echo use seem to be much more related to their positive self-image beliefs and their abilities, or concerns for remaining independent.

\section{Individual IVA Interactions in Shared Environments}

While we did encounter participants that were apathetic about news of "Alexa listening" [39,51], similar to other studies, privacy emerged as a significant concern and a reason for limiting use $[13,17,26,30]$. However, some participants' initial interests in using Echo, even for casual tasks or convenience, were sometimes limited by secondary household users due to privacy concerns or differing interaction needs. We found that privacy concerns of secondary users were consistent with that of the infrequent IVA users mentioned in Cowan's study of smart home devices [13]. However, unlike prior findings [13,51], secondary users in our study were much more aware of IVA privacy issues, less tolerant of potential privacy violations, and had more influence over the device's usage. Participants discussed initially changing their interactions with the Echo device noting practices similar to those mentioned by Zeng and colleagues (e.g., moving the device, limiting use to a specific room) $[13,51]$. However, this often ultimately led to abandonment. Our findings suggest a need for better support for shared use.

\section{IVA Use in Independent Living}

IVA and other conversational interfaces are found to be beneficial for supporting people with disabilities $[1,39]$. Our participants also saw the potential for current IVAs to support them if they had a change in ability or health status. Our findings build on prior work noting that participants with disabilities in our studies also found Echo useful for supporting independence $[1,39]$. Participants also saw the current functionality of the Echo as potentially useful for supporting wellness through music or companionship for older adults experiencing mental illness, social isolation, or a medical condition that limits mobility. While most IVA devices are not approved to access personal health data, Alexa is already being explored to help reduce social isolation in older adults [21], and there is emerging research examining IVA use for health and wellness $[5,8,40,41]$.

Participants saw the future potential for Echo to assist with caregiving tasks, finding and managing health-related information, and for improving wellness and human connections across the LPC. Participants discussed future uses of IVAs in the LPC, such as to support individual healing, wellness, and memory support as well as caregiving, socialization, and community health. However, similar to broader concerns about IVA use in health [5], there are open challenges for findings ways to design applications that are effective, but also safe and trustworthy. Most participants saw the greatest potential for IVAs in the
LPC was as a tool to help them better connect with people, resources, and the LPC community through better information access. Some features mentioned, such as the ability to call other residents are currently available in commercial IVAs; however, participants also discussed interaction difficulties of navigating lists by voice, which is still an open IVA design challenge [9]. Considering that in some larger LPCs such as the one in this study, there can be hundreds of users, it would also be useful to consider features that help users not only filter and customize contacts but also support the discovery of people with common interests. Other suggestions provided by participants included the use of an IVA to view and sign up for events, view menus, and find people and resources available to help with everyday tasks. Future work that explores ways to support human-to-human and human-toorganization connections in independent living may improve IVA acceptance and community engagement among older adults.

\section{LIMITATIONS}

Participants in our study were provided with an Echo and technical support in exchange for their involvement in an early adopters' program at the LPC. While participants in the program were volunteers that expressed interest in Echo, the program for some involved exploring potential Echo uses. Therefore, some users may not have become sustained users of Echo either way. Participants of the early adopters' program also had higher technology acceptance, average household incomes, and education levels, which may have influenced their usage and optimism about future use [34]. Participants' experiences with IVAs may be different from others that choose their device, have less support, or different socio-economical, educational, and technical literacy. We limited the size of our focus groups; however, another limitation of our work is that while useful for capturing broad perspectives, focus groups can limit the ability to gain deep, objective insights about individual perspectives. Data captured through other methods may lend additional insight on reasons for Echo use or non-use.

\section{CONCLUSIONS}

This paper reports findings from eight focus groups conducted at a multi-site LPC, where we explored reasons older adults use, do not use and limit their use of Echo. Findings indicate that over the span of a year, most residents who were early adopters became non-users due to challenges related to finding beneficial uses, beliefs about independence and Echo use, and use in shared spaces. We contribute a discussion of reasons for non-use and discuss opportunities for IVAs to better support aging, accessibility, and independent living in the future.

\section{ACKNOWLEDGEMENTS}

We want to thank the LPC staff and residents for sharing their experiences with deploying and using Echo. We thank the Indiana University EMPOWER grant program for supporting this research. We also thank those that provided feedback that helped us improve the paper. 


\section{REFERENCES}

1. Ali Abdolrahmani, Ravi Kuber, and Stacy M. Branham. 2018. "Siri Talks at You": An Empirical Investigation of Voice-Activated Personal Assistant (VAPA) Usage by Individuals Who Are Blind. In Proceedings of the 20th International ACM SIGACCESS Conference on Computers and Accessibility (ASSETS '18), 249-258. https://doi.org/10.1145/3234695.3236344

2. Richard P. Bagozzi. 2007. The Legacy of the Technology Acceptance Model and a Proposal for a Paradigm Shift. Journal of the Association for Information Systems; Atlanta 8, 4: 243-254.

3. Eric P.S. Baumer. 2015. Usees. In Proceedings of the 33rd Annual ACM Conference on Human Factors in Computing Systems (CHI '15), 3295-3298. https://doi.org/10.1145/2702123.2702147

4. Eric P.S. Baumer, Phil Adams, Vera D. Khovanskaya, Tony C. Liao, Madeline E. Smith, Victoria Schwanda Sosik, and Kaiton Williams. 2013. Limiting, Leaving, and (Re)Lapsing: An Exploration of Facebook Nonuse Practices and Experiences. In Proceedings of the SIGCHI Conference on Human Factors in Computing Systems (CHI '13), 3257-3266. https://doi.org/10.1145/2470654.2466446

5. Timothy W. Bickmore, Ha Trinh, Stefan Olafsson, Teresa K. O’Leary, Reza Asadi, Nathaniel M. Rickles, and Ricardo Cruz. 2018. Patient and Consumer Safety Risks When Using Conversational Assistants for Medical Information: An Observational Study of Siri, Alexa, and Google Assistant. Journal of Medical Internet Research 20, 9: e11510. https://doi.org/10.2196/11510

6. Ann Blandford, Dominic Furniss, and Stephann Makri. 2016. Qualitative Hci Research: Going Behind the Scenes. Morgan \& Claypool Publishers.

7. A.J. Bernheim Brush, Bongshin Lee, Ratul Mahajan, Sharad Agarwal, Stefan Saroiu, and Colin Dixon. 2011. Home Automation in the Wild: Challenges and Opportunities. In Proceedings of the SIGCHI Conference on Human Factors in Computing Systems (CHI '11), 2115-2124. https://doi.org/10.1145/1978942.1979249

8. Susana Buinhas, Ana Paula Cláudio, Maria Beatriz Carmo, João Balsa, Afonso Cavaco, Anabela Mendes, Isa Félix, Nuno Pimenta, and Mara Pereira Guerreiro. 2019. Virtual Assistant to Improve Self-care of Older People with Type 2 Diabetes: First Prototype. In Gerontechnology (Communications in Computer and Information Science), 236-248.

9. Leigh Clark, Nadia Pantidi, Orla Cooney, Philip Doyle, Diego Garaialde, Justin Edwards, Brendan Spillane, Emer Gilmartin, Christine Murad, Cosmin Munteanu, Vincent Wade, and Benjamin R. Cowan. 2019. What Makes a Good Conversation?: Challenges in Designing Truly Conversational Agents. 475. https://doi.org/10.1145/3290605.3300705
10. James Clawson, Jessica A. Pater, Andrew D. Miller, Elizabeth D. Mynatt, and Lena Mamykina. 2015. No Longer Wearing: Investigating the Abandonment of Personal Health-tracking Technologies on Craigslist. In Proceedings of the 2015 ACM International Joint Conference on Pervasive and Ubiquitous Computing (UbiComp '15), 647-658. https://doi.org/10.1145/2750858.2807554

11. Juliet Corbin, Anselm L. Strauss, and Anselm Strauss. 2015. Basics of Qualitative Research. SAGE.

12. J. F. Coughlin, L. A. D'Ambrosio, B. Reimer, and M. R. Pratt. 2007. Older Adult Perceptions of Smart Home Technologies: Implications for Research, Policy Market Innovations in Healthcare. In 2007 29th Annual International Conference of the IEEE Engineering in Medicine and Biology Society, 18101815. https://doi.org/10.1109/IEMBS.2007.4352665

13. Benjamin R. Cowan, Nadia Pantidi, David Coyle, Kellie Morrissey, Peter Clarke, Sara Al-Shehri, David Earley, and Natasha Bandeira. 2017. "What Can I Help You with?": Infrequent Users' Experiences of Intelligent Personal Assistants. In Proceedings of the 19th International Conference on Human-Computer Interaction with Mobile Devices and Services (MobileHCI '17), 43:1-43:12. https://doi.org/10.1145/3098279.3098539

14. Fred D. Davis. 1989. Perceived Usefulness, Perceived Ease of Use, and User Acceptance of Information Technology. MIS Quarterly 13, 3: 319-340. https://doi.org/10.2307/249008

15. George Demiris, Brian K. Hensel, Marjorie Skubic, and Marilyn Rantz. 2008. Senior residents' perceived need of and preferences for "smart home" sensor technologies. International Journal of Technology Assessment in Health Care 24, 1: 120-124. https://doi.org/10.1017/S0266462307080154

16. George Demiris, Marilyn Rantz, Myra Aud, Karen Marek, Harry Tyrer, Marjorie Skubic, and Ali Hussam. 2004. Older adults' attitudes towards and perceptions of "smart home" technologies: a pilot study. Medical Informatics and the Internet in Medicine 29, 2: 87-94. https://doi.org/10.1080/14639230410001684387

17. Aarthi Easwara Moorthy and Kim-Phuong L. Vu. 2014. Voice Activated Personal Assistant: Acceptability of Use in the Public Space. In Human Interface and the Management of Information. Information and Knowledge in Applications and Services (Lecture Notes in Computer Science), 324334.

18. Daniel A. Epstein, Monica Caraway, Chuck Johnston, An Ping, James Fogarty, and Sean A. Munson. 2016. Beyond Abandonment to Next Steps: Understanding and Designing for Life After Personal Informatics Tool Use. In Proceedings of the 2016 CHI Conference on Human Factors in Computing Systems (CHI '16), 1109-1113. https://doi.org/10.1145/2858036.2858045 
19. Shion Guha, Eric P.S. Baumer, and Geri K. Gay. 2018. Regrets, I'Ve Had a Few: When Regretful Experiences Do (and Don'T) Compel Users to Leave Facebook. In Proceedings of the 2018 ACM Conference on Supporting Groupwork (GROUP '18), 166-177. https://doi.org/10.1145/3148330.3148338

20. Sangyeal Han and Heetae Yang. 2018. Understanding adoption of intelligent personal assistants. Industrial Management \& Data Systems. https://doi.org/10.1108/IMDS-05-2017-0214

21. Christina Ianzito. Tech Like Alexa Might Help With Isolation, Loneliness. Retrieved October 2, 2019 from https://www.aarp.org/home-family/personaltechnology/info-2018/isolation-loneliness-technologyhelp.html

22. Jenny Kitzinger. 1995. Qualitative Research: Introducing focus groups. BMJ 311, 7000: 299-302. https://doi.org/10.1136/bmj.311.7000.299

23. Jarosไlaw Kowalski, Anna Jaskulska, Kinga Skorupska, Katarzyna Abramczuk, Cezary Biele, Wies\law Kopeć, and Krzysztof Marasek. 2019. Older Adults and Voice Interaction: A Pilot Study with Google Home. In Extended Abstracts of the 2019 CHI Conference on Human Factors in Computing Systems (CHI EA '19), LBW0187:1-LBW0187:6. https://doi.org/10.1145/3290607.3312973

24. Amanda Lazar, Christian Koehler, Joshua Tanenbaum, and David H. Nguyen. 2015. Why We Use and Abandon Smart Devices. In Proceedings of the 2015 ACM International Joint Conference on Pervasive and Ubiquitous Computing (UbiComp '15), 635-646. https://doi.org/10.1145/2750858.2804288

25. Yuting Liao, Jessica Vitak, Priya Kumar, Michael Zimmer, and Katherine Kritikos. 2019. Understanding the Role of Privacy and Trust in Intelligent Personal Assistant Adoption. In Information in Contemporary Society (Lecture Notes in Computer Science), 102113.

26. Irene Lopatovska, Alice London Griffin, Kelsey Gallagher, Caitlin Ballingall, Clair Rock, and Mildred Velazquez. 2019. User recommendations for intelligent personal assistants. Journal of Librarianship and Information Science: 0961000619841107. https://doi.org/10.1177/0961000619841107

27. Irene Lopatovska and Heyrling Oropeza. 2018. User interactions with "Alexa" in public academic space. Proceedings of the Association for Information Science and Technology 55, 1: 309-318. https://doi.org/10.1002/pra2.2018.14505501034

28. Irene Lopatovska, Katrina Rink, Ian Knight, Kieran Raines, Kevin Cosenza, Harriet Williams, Perachya Sorsche, David Hirsch, Qi Li, and Adrianna Martinez. 2019. Talk to me: Exploring user interactions with the Amazon Alexa. Journal of Librarianship and Information Science 51, 4: 984-997. https://doi.org/10.1177/0961000618759414
29. Irene Lopatovska and Harriet Williams. 2018. Personification of the Amazon Alexa: BFF or a Mindless Companion. In Proceedings of the 2018 Conference on Human Information Interaction \& Retrieval (CHIIR '18), 265-268. https://doi.org/10.1145/3176349.3176868

30. Ewa Luger and Abigail Sellen. 2016. "Like Having a Really Bad PA": The Gulf Between User Expectation and Experience of Conversational Agents. In Proceedings of the 2016 CHI Conference on Human Factors in Computing Systems (CHI '16), 5286-5297. https://doi.org/10.1145/2858036.2858288

31. Michal Luria, Guy Hoffman, and Oren Zuckerman. 2017. Comparing Social Robot, Screen and Voice Interfaces for Smart-Home Control. In Proceedings of the 2017 CHI Conference on Human Factors in Computing Systems (CHI '17), 580-628. https://doi.org/10.1145/3025453.3025786

32. Nora McDonald, Sarita Schoenebeck, and Andrea Forte. 2019. Reliability and Inter-rater Reliability in Qualitative Research: Norms and Guidelines for CSCW and HCI Practice. Proc. ACM Hum.-Comput. Interact. $\quad 3, \quad$ CSCW: 72:1-72:23. https://doi.org/10.1145/3359174

33. Anne-Sophie Melenhorst, Wendy A. Rogers, and Don G. Bouwhuis. 2006. Older adults' motivated choice for technological innovation: Evidence for benefit-driven selectivity. Psychology and Aging 21, 1: 190-195. https://doi.org/10.1037/0882-7974.21.1.190

34. Barbara Barbosa Neves, Fausto Amaro, and Jaime R. S. Fonseca. 2013. Coming of (Old) Age in the Digital Age: ICT Usage and Non-Usage among Older Adults. Sociological Research Online 18, 2: 22-35. https://doi.org/10.5153/sro.2998

35. Barbara Barbosa Neves, Rachel L. Franz, Cosmin Munteanu, Ronald Baecker, and Mags Ngo. 2015. "My Hand Doesn'T Listen to Me!": Adoption and Evaluation of a Communication Technology for the 'Oldest Old.' In Proceedings of the 33rd Annual ACM Conference on Human Factors in Computing Systems (CHI '15), 1593-1602. https://doi.org/10.1145/2702123.2702430

36. Pew Research Center. Voice assistants used by $46 \%$ of Americans, mostly on smartphones. Fact Tank: News in the Numbers. Retrieved September 18, 2019 from https://www.pewresearch.org/facttank/2017/12/12/nearly-half-of-americans-use-digitalvoice-assistants-mostly-on-their-smartphones/

37. Martin Porcheron, Joel E. Fischer, Stuart Reeves, and Sarah Sharples. 2018. Voice Interfaces in Everyday Life. In Proceedings of the 2018 CHI Conference on Human Factors in Computing Systems (CHI '18), 640:1-640:12. https://doi.org/10.1145/3173574.3174214

38. François Portet, Michel Vacher, Caroline Golanski, Camille Roux, and Brigitte Meillon. 2013. Design and evaluation of a smart home voice interface for the 
elderly: acceptability and objection aspects. Personal and Ubiquitous Computing 17, 1: 127-144. https://doi.org/10.1007/s00779-011-0470-5

39. Alisha Pradhan, Kanika Mehta, and Leah Findlater. 2018. "Accessibility Came by Accident": Use of Voice-Controlled Intelligent Personal Assistants by People with Disabilities. In Proceedings of the 2018 CHI Conference on Human Factors in Computing Systems (CHI '18), 459:1-459:13. https://doi.org/10.1145/3173574.3174033

40. A. Reis, D. Paulino, H. Paredes, I. Barroso, M. J. Monteiro, V. Rodrigues, and J. Barroso. 2018. Using intelligent personal assistants to assist the elderlies An evaluation of Amazon Alexa, Google Assistant, Microsoft Cortana, and Apple Siri. In 2018 2nd International Conference on Technology and Innovation in Sports, Health and Wellbeing (TISHW), 1-5. https://doi.org/10.1109/TISHW.2018.8559503

41. Jamie Sanders and Aqueasha Martin-Hammond. 2019. Exploring Autonomy in the Design of an Intelligent Health Assistant for Older Adults. In Proceedings of the 24th International Conference on Intelligent User Interfaces: Companion (IUI '19), 95-96. https://doi.org/10.1145/3308557.3308713

42. Christine Satchell and Paul Dourish. 2009. Beyond the user: use and non-use in HCI. In Proceedings of the 21st Annual Conference of the Australian ComputerHuman Interaction Special Interest Group on Design: Open $24 / 7 \quad$ OZCHI '09, 9. https://doi.org/10.1145/1738826.1738829

43. Alex Sciuto, Arnita Saini, Jodi Forlizzi, and Jason I. Hong. 2018. "Hey Alexa, What's Up?": A MixedMethods Studies of In-Home Conversational Agent Usage. In Proceedings of the 2018 Designing Interactive Systems Conference (DIS '18), 857-868. https://doi.org/10.1145/3196709.3196772

44. Neil Selwyn. 2003. Apart from technology: understanding people's non-use of information and communication technologies in everyday life. Technology in Society 25, 1: 99-116. https://doi.org/10.1016/S0160-791X(02)00062-3

45. Patrick C. Shih, Kyungsik Han, Erika Shehan Poole, Mary Beth Rosson, and John M. Carroll. 2015. Use and Adoption Challenges of Wearable Activity Trackers. Retrieved November 18, 2019 from https://www.ideals.illinois.edu/handle/2142/73649

46. D. B. Smith. 1990. Human factors and aging: an overview of research needs and application opportunities. Human Factors 32, 5: 509-526. https://doi.org/10.1177/001872089003200502

47. Michel Vacher, Benjamin Lecouteux, Dan Istrate, Thierry Joubert, François Portet, Mohamed Sehili, and Pedro Chahuara. Experimental Evaluation of Speech Recognition Technologies for Voice-based Home Automation Control in a Smart Home.

48. Viswanath Venkatesh, Michael G. Morris, Gordon B. Davis, and Fred D. Davis. 2003. User Acceptance of
Information Technology: Toward a Unified View. MIS Quarterly 27, 3: 425-478. https://doi.org/10.2307/30036540

49. Linda Wulf, Markus Garschall, Julia Himmelsbach, and Manfred Tscheligi. 2014. Hands Free - Care Free: Elderly People Taking Advantage of Speech-only Interaction. In Proceedings of the 8th Nordic Conference on Human-Computer Interaction: Fun, Fast, Foundational (NordiCHI '14), 203-206. https://doi.org/10.1145/2639189.2639251

50. Wyatt, S.M.E., Oudshoorn, N., Pinch, T., and ASCoR (FMG). 2003. Non-users also matter: The construction of users and non-users of the Internet. In Now Users Matter: The Co-construction of Users and Technology. MIT Press, 67-79. Retrieved August 6, 2019 from https://dare.uva.nl/personal/pure/en/publications/nonus ers-also-matter-the-construction-of-users-andnonusers-of-the-internet(9fc15327-9657-4e65-9af3b5def673f4e9).html

51. Eric Zeng, Shrirang Mare, and Franziska Roesner. 2017. End User Security and Privacy Concerns with Smart Homes. 65-80. Retrieved September 5, 2019 from https://www.usenix.org/conference/soups2017/technic al-sessions/presentation/zeng 